\title{
Théorie parétienne des élites et moment machiavélien
}

Paretian theory of the elites and Machiavellian moment

\section{Claire Baldin et Ludovic Ragni}

\section{(2) OpenEdition \\ 1 Journals}

Édition électronique

URL : http://journals.openedition.org/ress/3597

DOI : $10.4000 /$ ress.3597

ISBN : 1663-4446

ISSN : $1663-4446$

Éditeur

Librairie Droz

Édition imprimée

Date de publication : 30 novembre 2016

Pagination : $219-249$

ISSN : 0048-8046

Référence électronique

Claire Baldin et Ludovic Ragni, "Théorie parétienne des élites et moment machiavélien », Revue européenne des sciences sociales [En ligne], 54-2 | 2016, mis en ligne le 30 novembre 2019, consulté le 04 janvier 2020. URL : http://journals.openedition.org/ress/3597 ; DOI : 10.4000/ress.3597 


\title{
THÉORIE PARÉTIENNE DES ÉLITES ET MOMENT MACHIAVÉLIEN
}

CLAIRE BALDIN \& LUDOVIC RAGNI

Université Côte d'Azur, Nice - CNRS - GREDEG

baldin@gredeg.cnrs.fr / ragni@unice.fr

\begin{abstract}
Résumé. Cet article explique comment la dynamique de l'équilibre social chez Pareto repose sur le principe de rupture du moment machiavélien. II resitue ce principe en termes d'équilibre entre virtù et fortuna puis en apprécie la transposition à l'étude de la dynamique de l'équilibre social parétien. Après avoir réévalué la méthode des approximations successives et de synthèse à laquelle Pareto recourt, nous montrons que le principe de rupture du moment machiavélien implique que l'explication de la dynamique sociale repose sur des lois tendancielles qui supposent de remettre en cause la synthèse des résultats de Pareto.
\end{abstract}

Mots-clés: histoire de la pensée économique, Nicolas Machiavel, méthodologie, Vilfredo Pareto.

\begin{abstract}
This article explains how the dynamic of Pareto's social equilibrium is based on the principle of rupture of the Machiavellian moment. The article sets out this principle in terms of equilibrium between virtù and fortuna, and then assesses the study of the transposition of the dynamics of Paretian social equilibrium. After having assessed the method of the successive approximations and synthesis used by Pareto, we show that the principle of rupture of the Machiavellian moment involves taking into consideration that the explanations of the dynamics of social equilibrium rest on tendency laws that call into question the synthesis of Pareto's results.
\end{abstract}

Keywords: history of economic thought, Niccolò Machiavelli, methodology, Vilfredo Pareto.

Codes JEL: BI30, BI5, B4I, Z00. 
Elle [la ploutocratie] a imaginé d'innombrables expédients, comme les énormes dettes publiques qu'elle sait bien ne pas pouvoir payer en définitive, comme ponction sur le capital, les impôts qui diminuent et épuisent les revenus de ceux qui ne spéculent pas,

les lois somptuaires dont l'histoire a déjà tant de fois démontré la vanité, et d’autres mesures semblables qui ont pour but de tromper les foules. (Pareto, I970 [1920], p. 57).

Deux raisons justifient l'appartenance de Pareto au courant machiavélien. L'une résulte du constat selon lequel Pareto développe une explication de la dynamique de l'équilibre social qui renvoie aux concepts de fortuna et de virtù auxquels Machiavel a recours pour expliquer les luttes de pouvoir politique. Cette transposition apparaît dans les interactions que Pareto prône entre les principales catégories de sa sociologie. Ainsi, pour Aron (1938-1940a, b, c et d; 1967), Burnham (1949 [1943]), Busino (1984), Audier (2005) ou Valade (2005) les catégories parétiennes de résidus, dérivations, intérêts et hétérogénéités sociales renvoient à une même conception de la nature humaine que celle décrite par Machiavel. Témoignant de cet emprunt, Pareto cite 70 fois Machiavel dans le Traité de sociologie générale. Lautre raison découle du constat que les catégories que les deux auteurs développent relèvent d’une méthodologie positive, et on ne peut plus réaliste, consistant à apprécier l'action humaine à partir des uniformités qu'ils en constatent dans l'histoire politique, économique et sociale. Pareto se réfère ici à Machiavel pour préciser sa propre méthodologie:

Celui qui voit objectivement les faits, celui qui ne veut pas, de propos délibéré, fermer les yeux à la lumière, est tout de même obligé de reconnaître que ce n'est pas en étant des moralistes timorés que les gouvernants font prospérer les nations; mais il passe le fait sous silence, ou s'excuse de l'imprimer, et accuse les évènements la «corruption» des mœurs. Pourtant, même ainsi, il n'évite pas le reproche d'immortalité dont on gratifia Machiavel, parce qu'il devait simplement exprimer des uniformités que tout le monde peut vérifier dans l'histoire. On l'a accusé d’avoir plagié Aristote et d'autres auteurs: il s'est simplement rencontré avec ceux qui ont décrit la réalité (Pareto, 1968 [1916], p. 1253-1254).

L'intention de Machiavel est en effet d'expliquer l'évolution politique des Principautés et des Républiques à partir des rapports que les peuples entretiennent avec leurs gouvernants et qu'il constate dans l'histoire des sociétés. Il cherche à expliquer, sous quelles conditions un régime politique perdure 
lorsqu'un équilibre advient entre virtù et fortuna et pour quelles raisons un régime politique peut en supplanter un autre lorsque cet équilibre est rompu. La première situation correspond à ce qu'il est convenu d'appeler le moment machiavélien, la seconde à sa rupture (Pocock, 1975).

De son côté, l'objectif de Pareto (1968 [1916]) est d'expliquer, à partir des luttes entre élites pour le pouvoir, comment les équilibres sociaux s'établissent ou sont rompus. Dans ce but, il propose de réaliser la synthèse des résultats qu'il met en évidence pour l'économie et la sociologie. Ces résultats sont supportés par la dialectique entre les catégories précédentes qui servent à déterminer les conditions prévalant aux états successifs des institutions économiques et sociales.

Dans ce cadre, cet article a pour objet d'apprécier comment la dynamique de l'équilibre social chez Pareto repose sur une série d'emprunts au principe de rupture du moment machiavélien. Nous montrons pourquoi ce principe rend difficile la synthèse que Pareto propose de ses résultats d'économie et de sociologie. Dans une première partie (I), après avoir resitué le principe de rupture du moment machiavélien en termes de déséquilibre entre virtù et fortuna (I.I), nous examinons sa transposition à l'étude de la dynamique de l'équilibre social parétien (I.2). Dans une seconde partie (2) nous évaluons pourquoi cette analyse ne permet pas la synthèse des résultats que Pareto cherche à établir. Après avoir apprécié le corpus méthodologique auquel renvoie la méthode de Pareto (2.I), nous montrons que le principe de rupture du moment machiavélien implique de considérer les explications de la dynamique sociale comme des lois tendancielles. Cette difficulté remet en cause la synthèse des résultats de Pareto (2.2). Nous illustrons notre propos à partir de la lutte entre élites partisanes du libre-échange et celles favorables au protectionnisme et les explications que Pareto propose de l'évolution des régimes politiques. 


\section{LA RUPTURE DU MOMENT MACHIAVÉLIEN COMME PRINCIPE DE LA DYNAMIQUE DE L'ÉQUILIBRE SOCIAL PARÉTIEN}

Il existe deux lectures de l'œuvre de Machiavel. L'une, fait une place importante au Prince (I532) comme manuel de l'art de gouverner avec cynisme. L'autre, accorde une place plus large au Discours sur la première décade de Tite-Live (I53I) et fait l'éloge du républicanisme ce qui n’empêche pas d’apprécier également le Prince comme un ouvrage écrit pour défendre la République (Strauss, I982 [1958]), même si Machiavel y dispense les conseils à un tyran pour maintenir le peuple à distance. Ces lectures attachent une importance particulière aux luttes entre les élites pour le pouvoir et aux relations qu'elles doivent entretenir avec le peuple pour maintenir l'équilibre social. Elles font donc une place importante à la rupture du moment machiavélien pour expliquer l'avènement ou le déclin des Républiques et des Principautés dont nous cernerons les implications chez Pareto.

I.I. VIRTÜ ET FORTUNA SOURCES DU MOMENT MACHIAVELIEN ET DE SA RUPTURE

À suivre Pocock (1975), deux interprétations du moment machiavélien sont envisageables.

La première renvoie à la période de l'histoire de l'Italie observée par Machiavel. Selon Bergès (2000), cette histoire est caractérisée par des circonstances politiques, intellectuelles, sociales et religieuses particulières qui ont conduit Machiavel à rédiger ses écrits politiques. Dans ce contexte, le concept de «moment machiavélien » désigne la cristallisation de la pensée de Machiavel sur les menaces qui pèsent sur la république de Florence en raison du désengagement participatif de ses citoyens pour sa survie. Pour autant, l'ouvrage de Pocock ne consacre à ce thème que 60 pages sur 360. L’auteur préfère défendre l'idée qu'il existe une pensée concurrente à la pensée politique classique lockienne, que l'on retrouve dans le républicanisme de Machiavel et dans la tradition républicaine nord-américaine. Sans engager une critique de cet ouvrage (voir Gaille-Nikodimov, 200I ; Ménissier, 200I) nous nous référerons à une analyse plus commune du moment machiavélien qui est promue par 
Pareto afin d'identifier ce qu'il y a de constant dans les rapports politiques et sociaux pour en dégager les lois d'évolution.

La seconde conception du moment machiavélien (Burnham, I949 [1943]; Strauss, 1982 [1958]; Colonna d'Istria et Frapet, 1980; Sfez, 2003; Aubier, 2005 ; Collin, 2008) concerne les interactions entre les concepts de virtù et de fortuna pour expliquer la gestion des affaires publiques. Pour Machiavel, la virtù permet de caractériser un ordre institutionnel (ordine) capable de résister aux assauts de la fortuna considérée comme source de hasard malveillant. La dynamique entre virtù et fortuna supporte ainsi les lois qui régissent l'évolution des institutions politiques, c'est-à-dire leurs moments d'équilibre et de rupture possibles. L'auteur du Prince en cherche confirmation dans la Rome antique et la Florence de son temps. De son côté, Pareto s'y réfère pour définir les catégories de sa sociologie afin d'expliquer la dynamique de l'équilibre social à partir des luttes que se livrent les élites pour le pouvoir en se référant à une même conception de la nature humaine que Machiavel: «les hommes sont rarement ou tout à fait bons ou tout à fait mauvais; mais et méchants, et bons, et médiocres s'accordent tous à ménager un Prince puissant, juste et habile» (Pareto, 1968 [1916], p. I037-I253).

Pareto reconnait également que Machiavel a toujours voulu dégager les uniformités qui existent entre les faits afin d'en déduire une explication des luttes entre élites : «Pour autant, même ainsi, il n’évite pas le reproche d'immortalité dont on gratifia Machiavel, parce qu'il avait simplement exprimé des uniformités que tout le monde peut vérifier dans l'histoire » (Pareto, ibid., p. I253, souligné par nous).

En effet pour Machiavel :

Quiconque compare le présent et le passé, voit que toutes les cités, tous les peuples ont toujours été et sont toujours animés des mêmes désirs, des mêmes passions. Ainsi, il est facile, par une étude exacte et bien détaillée du passé, de prévoir dans une République ce qui va arriver et alors il faut se servir des moyens mis en usage par les anciens ou, n'en trouvant pas d'usités, en imaginer de nouveaux, d'après les ressemblances des évènements (Machiavel, 1952, p. 467 - Discours...). 
Dans ce contexte, la fortuna, comme entité personnifiée - déesse de la fertilité mais aussi de la force arbitraire et de l'instabilité - supporte une double signification. Elle désigne dans Le Prince ce que les hommes ne doivent pas à leur mérite personnel (Lang, 2003, p. 650). Elle est synonyme de bonne fortune et de ce que certains hommes politiques doivent à d'autres (Machiavel, 1952, p. 306-312-Le Prince). Elle exprime aussi l'incertitude comme l'ensemble des menaces qui planent sur la stabilité de tout régime politique. Elle est synonyme de hasard malveillant comme source d'instabilité sociale et de lutte pour le pouvoir. Elle désigne ce que les hommes doivent à la fortune du hasard et qu’ils doivent savoir saisir pour conserver ou accéder au pouvoir. Ainsi, la fortuna est source de menaces pour les hommes qui doivent agir pour le maintien de la République, y compris en recourant à la force, s'ils sont marqués par la virtù. Les menaces de la fortuna peuvent permettre à ceux qui possèdent les qualités requises (virtù) de conquérir le pouvoir ou de s'octroyer des avantages. Elle est en ce sens à l'origine d'instants de déstabilisation des équilibres sociaux comme autant de moments de luttes pour le pouvoir. Entre fortune et infortune, il y a donc place pour l'action de ceux qui désirent s'approprier le pouvoir contre ceux qui veulent le conserver. Dans le Traité, Pareto transpose cette dynamique à l'étude des luttes entre les élites pour le pouvoir. Il en propose une analyse à partir des raisons qui motivent l'action des hommes quand leur but est de promouvoir un régime politique ou une institution.

Machiavel a voulu traiter d'un cas concret, qui devient aussi un cas particulier de la question générale. Il a écrit Le Prince, mais exactement sur le même modèle, il aurait pu écrire Les Républiques. Il l’a fait en partie dans les Discours sur la première décade de Tite-Live, et s'il avait vécu de nos jours, il aurait pu faire porter ses études sur les Régimes parlementaires. Il a recherché quels sont les moyens les plus appropriés pour que les princes conservent le pouvoir, et il a examiné les deux hypothèses, du prince nouveau et du prince héréditaire. Sur le même modèle, il aurait pu faire des recherches analogues pour les autres régimes politiques (Pareto, 1968 [1916], p. 1254-1255). 
De son côté, la virtù désigne les valeurs physiques et morales des hommes. Elle recouvre les qualités qui leur permettent de saisir les occasions de l'histoire afin, soit d'accéder au pouvoir lorsque la fortuna les y incite, soit de sauvegarder les institutions s'ils se révèlent capables de résister à ses affres. C'est donc grâce à la virtù du dirigeant et au partage des valeurs civiques parmi les citoyens (ordine) que la République peut perdurer. La virtù désigne les qualités que le Prince ou les hommes doivent posséder pour se maintenir au pouvoir ou permettre la survie des institutions. Pour garantir une institution ou un régime politique, la dynamique entre virtù et fortuna implique que l'éthique privilégie la fin et que le bien commun soit dissocié des moyens nécessaires à son maintien. En ce sens, le Machiavel machiavélique de la «légende noire» ne correspond pas à celui du penseur de la raison d’État. Machiavel est républicain même si ses préceptes s'appliquent à d’autres institutions (Pocock, 1975). Pour autant, il ne croit pas aux régimes d’assemblées qui légifèrent parce qu'il considère qu'ils ne sont pas viables comme le lui confirme l'histoire de Florence. Ce point de vue n'échappe pas aux critiques que Pareto porte aux régimes ploutocratiques:

les parlements modernes paraissent être un instrument efficace de la ploutocratie démagogique. D’abord dans les élections, ensuite dans les délibérations, ils offrent un large champ à l'activité des hommes qui ont un fort instinct de combinaisons. C’est pourquoi le régime parlementaire moderne suit en partie le sort de la ploutocratie: il prospère, et décline avec elle; et ses transformations que l'on appelle aussi transformation de la démocratie, s'accompagnent de la vicissitude de la ploutocratie (Pareto, 1970 [1920], p. 50).

Dès lors, pour Machiavel à propos des principautés (Machiavel, I952, p. 317-320 - Le Prince), et pour Pareto à propos des ploutocraties, l'organisation politique suppose que certaines factions cherchent à en dominer d'autres en exerçant leur gouvernement. Elle suppose aussi que ces mêmes factions cherchent à dominer le peuple qui désire ne pas l'être.

Car en toute cité on trouve ces deux humeurs différentes, desquelles la source est que le populaire n'aime point tant être commandé ni opprimé des plus gros. Et les gros ont envie de commander et opprimer le peuple. Et de ces deux différents appétits s'élèvent dans les villes un de ces trois effets : ou Principauté ou liberté ou licence (ibid., p. 317). 
On retrouve cette opposition entre les masses et les élites chez Pareto pour qui une élite est définie comme l'ensemble des personnes qui possèdent certaines qualités contre celles, plus nombreuses, qui ne les possèdent pas. De son côté, Machiavel reconnaît l'existence d'un incessant mouvement de déstabilisation de la virtù par la fortuna auquel correspond un mouvement entre ceux qui ne désirent pas être gouvernés et ceux qui ont l'intention de diriger. L'avenir de la République ou d’une institution politique dépend donc du partage vertueux des valeurs civiques entre les citoyens et le Prince ou entre les citoyens et l'élite. Les rapports entre fortuna et virtù impliquent de reconnaître qu'il existe deux interprétations complémentaires de leur dynamique. L’une accorde une place prépondérante à la fortuna comme source d'incertitude et d’instabilité qui détermine les lois de la dynamique sociale. L’autre retient que cette dynamique résulte des rapports entre les deux concepts. Dans les deux cas, l'évolution politique s'organise comme une suite de ruptures du moment machiavélien dont Pareto s'inspire pour exprimer les lois de l'équilibre social entre élites politiques et masse des gouvernés.

\section{I.2. LES CATÉGORIES DE LA SOCIOLOGIE PARÉTIENNE SOURCE DE RELECTURE DU MOMENT MACHIAVÉLIEN}

Pour Pareto, l'équilibre social et ses ruptures résultent des luttes entre élites politiques d'une part, et entre les élites et l'ensemble des individus d'autre part. Une classe d'individus, ou hétérogénéité sociale dans la terminologie parétienne, est caractérisée par une élite constituée de quelques individus aux qualités exceptionnelles et par une majorité aux qualités moindres. Les luttes entre élites gouvernementales ou non-gouvernementales supportent les mécanismes qui prévalent à la conquête du pouvoir ou à la défense d'une institution (ibid.,p. I297-I30I). Elles permettent d'identifier une suite de moments machiavéliens dont les dynamiques résultent des interactions entre les catégories de résidus, dérivations, intérêts ou hétérogénéité et circulation sociale qui supportent les actions économiques et sociales des individus et des groupes (ibid., p.ı307-I308). Ainsi, en référence au concept d’actions non-logiques, Pareto considère que les comportements des acteurs sociaux dépendent des forces psychologiques qui échappent à leur contrôle conscient et 
qui se manifestent de manière fatale dans la catégorie des résidus. Ceux-ci sont l'expression indirecte des sentiments et des instincts propres à la nature humaine (Perrin, 1966 ; Busino, 1999 ; 2008) que l'on retrouve chez Machiavel. Impossibles à appréhender directement, ils sont source des connaissances communes qui organisent la partie constante des actions non-logiques afin d'adapter les acteurs sociaux à leur environnement (Pareto, 1968 [1916], p. 452-466).

Les dérivations, comme partie variable de ce qui est exprimé, supportent les mécanismes de rationalisation que les individus déploient pour justifier leurs actions logiques ou non-logiques selon les circonstances. Elles sont l'expression de raisonnements, exacts ou erronés, qui permettent aux individus de se convaincre ou de convaincre les autres de la pertinence de leurs analyses ou des idéologies qu'ils veulent promouvoir avec cynisme ou bonne foi (ibid., p. 789-790). À ce titre, elles sont ce en quoi l'acteur social à de bonnes raisons de croire pour justifier ses actes (Boudon, 2000 ; 20I3). Pour une autre part, les dérivations résultent des résidus qui s'expriment par leur intermédiaire.

Les résidus, de manière instinctive, et les dérivations comme argumentations pseudo-logiques, expriment les intérêts des acteurs sociaux qui recouvrent les désirs de possession de biens et de pouvoir résultant de l'activation d’actions non-logiques et, pour une moindre part, d'actions logiques (Pareto, 1968 [1916], p. I280).

Les relations de dépendances entre ces catégories supportent les luttes de pouvoir auxquelles les élites se livrent. Elles sont l'expression de dérivations et de résidus différents qui caractérisent une élite contre une autre (ibid., p. 78-80, p. I293-I350). Cette dialectique de lutte entre élites permet à Pareto d'expliquer l'évolution des équilibres sociaux-politiques comme une loi ondulatoire dont les retournements peuvent être identifiés comme autant de ruptures du moment machiavélien. Leur expression est exprimée au travers des formes prises par la catégorie de l'hétérogénéité et circulation sociale pour rendre compte de la circulation des élites.

À la suite d'Aron (1938-1940b, 1967) on peut réduire la typologie des «résidus » à leurs classes I et 2 pour expliquer cette dynamique. Ces résidus cristallisent, à notre avis, les moments machiavéliens où les forces nécessaires au renouvellement ou au maintien de certaines élites s'opposent. Ainsi, pour se 
maintenir au pouvoir en fin de cycle, une élite doit être guidée par davantage de résidus d'instinct de combinaison (classe I) contre ceux de persistance des agrégats dont elle manquerait (classe 2). Les résidus de classe I supportent des instincts de ruses, d’adaptation ou d'innovation. L'élite au pouvoir est souvent amenée à activer ces résidus afin d'éviter, s'ils se révèlent suffisants, de recourir à la force contre une élite montante. Les instincts de combinaison correspondent clairement aux comportements de ruse que Machiavel prête aux Renards. Ceux de la classe 2 sont davantage tournés vers la défense violente d'une institution ou vers sa conquête. Ils favorisent les comportements de défense de la famille, de la religion ou de la Nation. Ils renvoient aux comportements des Lions de Machiavel au sens où, si une élite désire se maintenir au pouvoir, elle doit être prête à recourir à la force. L'élite au pouvoir peut aussi, en activant les résidus de la classe I, s’assurer la fidélité d'individus disposant de résidus de classe 2 afin qu'ils écartent, éventuellement par la force, ceux qui voudraient conquérir le pouvoir s'ils disposent de résidus des classes I et 2 en proportion idoine. La dynamique entre élites politiques dépend également des dérivations comme ensemble des arguments auxquels les acteurs sociaux recourent pour défendre leurs intérêts. Dans ce cadre, les dérivations sont l'expression de raisonnements pseudologiques, souvent idéologiques, auxquels les acteurs ont de bonnes raisons de croire sans que les raisons que ces acteurs invoquent puissent être considérées comme toujours logiques ou parfaitement rationnelles.

Il fait peu de doute que les catégories de Pareto empruntent largement aux thèmes machiavéliens de la force et de la ruse (Burnham, 1949 [1943]). L’auteur du Traité se réfère en effet à celui du Prince, en indiquant que l'opinion de certaines élites finit par s'imposer par la ruse quand les dérivations qu’elles auront activées se révèleront efficaces pour convaincre du bien-fondé de leurs objectifs. En d'autres circonstances, c'est le recours à la force qui permet à une élite d'exercer le pouvoir ou de défendre une institution. Reconnaître que l'homme est soumis à certains instincts, pouvant se révéler tout à la fois bons et mauvais, c'est reconnaître qu'il peut être soumis aux résidus d'instinct de combinaisons et de persistance des agrégats. Pareto montre combien les stratégies qui reposent sur les résidus de classe I, si elles permettent à court terme la survie d'une élite, conduisent souvent à sa 
perte si cette même élite refuse de recourir à la force. La dynamique entre résidus d'instinct de combinaisons et de persistance des agrégats laisse donc place aux rapports de forces que Machiavel exprime par la métaphore des Renards, porteurs d'instinct des combinaisons, et des Loups porteurs de persistance des agrégats. Elle s'exprime aussi dans le fait que les Renards recourent à la ruse pour se garantir la force des Lions afin de répondre à celle des Loups qui voudraient s'emparer du pouvoir.

Ainsi, savoir activer au bon moment les dérivations efficaces peut s'interpréter comme savoir exprimer les principes de virtù nécessaires à l'exercice d'un pouvoir efficace selon Machiavel. De même, la fortuna, comme synonyme de conditions à saisir, caractérise les moments d'opposition entre élites parétiennes aux virtù différentes lorsqu'il s'agit pour Machiavel d'expliquer, soit comment s'organise une institution pour défendre l'ordre établi face aux assauts d'une élite montante, soit comment une élite peut réduire cet ordre pour conquérir le pouvoir. En ce sens, les préceptes qui prévalent à la rupture de l'équilibre social selon Pareto relèvent du même mode d'explication que ceux que Machiavel préconise. Pareto explique en effet comment les luttes entre élites caractérisées par des quantités différentes de résidus des classes I et 2, conduisent à la réduction ou à l'émergence d'un régime politique. Pour lui, c'est l'accumulation d'individus possédant des résidus d'instinct de combinaisons parmi la classe dominante qui explique la perte de leur pouvoir parce qu'ils préfèreront la ruse consistant à acheter la paix pour pérenniser leurs intérêts à court terme contre le fait de préserver leur pouvoir par la force. Cette dynamique pour la conquête du pouvoir se double d'une dynamique entre élites politiques détentrices de ce même pouvoir et la masse du peuple. Une élite politique ne peut se maintenir au pouvoir que si elle détient suffisamment de résidus de persistance des agrégats pour s'imposer à la masse en recourant à l'argumentation idéologique et, éventuellement, à la force. Ainsi, en se référant à Machiavel, Pareto insiste sur le rôle des résidus de deuxième classe qui font défaut dans l'Italie du début du Xxe siècle (Pareto, I968 [1916], p. 1685). De même, citant les Discours, il indique que pour conserver un État, le Prince trouvera moins d'obstacles chez les peuples grossiers soumis à la religion que chez les peuples civilisés qui sont par nature corrompus. Il rejoint ici Machiavel pour qui la virtù du Prince, s'il 
veut conserver le pouvoir, doit faire en sorte que perdure un ordine qui répond aux intérêts du peuple. En termes parétiens, il s'agit d'élaborer des dérivations idoines permettant à une élite de s'imposer pour garantir un équilibre durable.

Néanmoins on voit par expérience de notre temps que ces Princes se sont faits grands qui n'ont pas tenu grand compte de leur foi, et qui ont su par ruse circonvenir l'esprit des hommes, et à la fin ils ont surpassé ceux qui se sont fondés sur la loyauté. Il faut donc savoir qu'il y a deux manières de combattre, l'une par les lois, l'autre par la force : la première sorte est propre aux hommes, la seconde propre aux bêtes; mais comme la première bien souvent ne suffit pas il faut recourir à la seconde. [...] Puis donc qu'un Prince doit savoir bien user de la bête, il en doit choisir le renard et le lion; car le lion ne se défend pas des rets, le renard des loups. Il faut donc être renard pour connaître les filets, et lion pour faire peur aux loups (Machiavel, 1952, p. 34I - Le Prince).

Si cet équilibre est rompu une autre élite accède au pouvoir.

\section{RUPTURE DU MOMENT MACHIAVÉLIEN ET PRINCIPE DE L'ÉQUILIBRE SOCIAL Une limite à la méthode des approximations successives}

L’objectifle plus achevé de Pareto est de mettre au jour les lois qui président au passage d'un équilibre social à un autre et non de considérer la sociologie comme l'étude de l'évolution de la société (Pareto, 1967 [1905-1918]; Busino, 1984). Après avoir resitué la méthodologie de Pareto nous en préciserons les limites à partir de deux exemples où intervient la rupture du moment machiavélien.

\section{I. LES RÉFÉRENTS MÉTHODOLOGIQUES PARÉTIENS}

Pareto (1987 [1898], 1968 [1916], I976 [1918]) propose de réaliser la synthèse de ses résultats d'économie et de sociologie en allant du simple au complexe ce qui pose des difficultés parce qu'il applique à ces disciplines des méthodologies difficiles à unifier même s'il considère qu'il convient de toujours procéder de manière logico-expérimentale (Marchionatti et Gambino, 1997; Marchionatti, 1999; Bruni, 2002). Pour autant, il recourt davantage à l'exemplification en sociologie qu’à l'expérimentalisme (Halbwachs, 1978 [1918]). 
L'étude de l'évolution des phénomènes économiques dans les temps voisins du nôtre et dans des sociétés qui ne diffèrent pas énormément de la nôtre est beaucoup plus utile que celle de leur origine [...] Elle nous permet d'abord de remplacer l'expérience directe, qui est impossible dans les sciences sociales [...] L'étude de l'évolution des phénomènes peut ensuite nous être utile en ce qu'elle nous facilite la découverte des uniformités que peut présenter cette évolution (Pareto, I966 [1906], p. 25-26).

Pareto use en fait de deux méthodes distinctes. L'une, propre à l'économie pure, consiste à admettre certaines hypothèses pour établir un raisonnement déductif afin de démontrer des théorèmes qui devraient ensuite être vérifiés :

je suis la voie de presque tous les économistes et je pose certaines entités avant les faits, au lieu de poser les faits avant les abstractions que représentent ces entités. Ce qui équivaut à détourner la méthode expérimentale pure. [...] Cela ne signifie pas qu'un ouvrage perde son caractère essentiellement expérimental s'il passe de l'abstraction à la représentation des faits mais cela signifie que, pour garder ce caractère, il est nécessaire que l'abstraction reste une simple hypothèse (Pareto, 1976 [1918], p. 104-105).

L'autre, propre à la sociologie et à l'économie appliquée, suppose de compiler des données et des exemples historiques afin d'établir des moyennes ou des corrélations (Pareto, ibid., p. I06-I07). Ces données permettent de circonscrire des lois qui servent à l'élaboration de raisonnements dont les résultats doivent être confirmés (ibid., p. I05). De ce point de vue, l’expérimentalisme de Pareto est tronqué parce qu'il suppose, soit de considérer l'observation des faits suffisante pour émettre une hypothèse mais sans la tester, soit d'induire des uniformités sans réaliser d'expériences. Il assimile en fait les uniformités observées à des lois tendancielles qui prennent la forme de séries statistiques en économie appliquée ou d'observations grossières en sociologie. Elles sont ainsi répertoriées dans la Gazette de Lausanne ou prises dans les grands textes historiques en référence notamment à Machiavel. Pareto explicite ainsi comment la circulation des élites résulte des interactions entre résidus, dérivations, intérêts et hétérogénéité sociale pour expliquer comment l'équilibre social advient ou est rompu. Cette explication doit permettre la synthèse des résultats d'économie pure et appliquée afin d'en élargir la pertinence en sociologie. 
Les résultats auxquels [l'économie] arrive constituent une partie intégrante et très importante de la sociologie; mais ils n'en constituent qu'une partie, qui, en certains phénomènes, peut être petite, négligeable, et qui, en tout cas, doit être combinée avec les autres parties, pour nous donner l'image des phénomènes concrets (Pareto, 1968 [1916], p. I282-1283).

C'est aux différentes branches des sciences sociales et à leur synthèse, exprimée par la sociologie, que nous pouvons demander la solution de tels problèmes (ibid., p. 1290).

La définition des champs de l'économie et de la sociologie repose sur la différence que Pareto prône entre actions logiques et non-logiques. L'économie est le champ des actions logiques qui répondent au principe selon lequel les buts que les agents poursuivent subjectivement sont identiques aux buts objectivement définis par le scientifique. Sont logiques les actions de l'ingénieur et de l'homo-øconomicus parce qu'ils sont capables de calculs rationnels ou de recourir à certains instruments pour atteindre l'objectif qu'ils recherchent intentionnellement. Le champ de la sociologie concerne les actions non-logiques et, dans une moindre mesure logiques (Pareto, 1967 [1905-19I8] , p. 8). Une action est non-logique si son but objectif diffère de son but subjectif indépendamment de l'existence ou non de tels buts. La méthode des approximations successives préconise ainsi que l'économie et la sociologie sont des disciplines complémentaires et que la sociologie est une discipline englobante de l'économie (Legris et Ragni, 1999), ce qui rend, à notre avis, problématique la synthèse des résultats des deux disciplines.

Pareto propose en effet, en première approximation, de démontrer les théorèmes de l'économie pure en recourant à un argumentaire hypothéticodéductif. Une fois cette étape achevée, il place au second plan les théorèmes obtenus parce qu'ils ne tiennent pas compte de la complexité des actions nonlogiques pour approcher la réalité (Pareto, 1966 [1906], p. 4I-48). En économie pure, ces théorèmes reposent sur les régularités comportementales des agents exprimées par les actions logiques (Pareto, 1987 [1898], p. 107). 
Considérée de ce seul point de vue, l'expérimentalisme correspond selon l'appréciation même de Pareto à la méthode déductive concrète qu'il emprunte à John Stuart Mill (1988 [1843], t.I, p. 509-521 ; t. 2, p.488-508). Pour Pareto, cette méthode: «est celle utilisée en science physique, et que Mill a décrite précisément sous la dénomination de méthode déductive concrète. De certains principes généraux, tirés de l'expérience, on déduit des théories qui ensuite sont vérifiées » (Pareto, 1966 [1898], p. 156-I57). Or, si Pareto se réfère à la méthode déductive concrète, il en dénature la première étape pour l'économie pure. En effet, pour Mill: «la méthode déductive concrète, contre celle purement déductive de la géométrie, suppose que les résultats dépendent de déductions établies en tenant compte de plusieurs causes (prémisses) qu'il faut analyser ensembles ou séparément» (Mill, I988 [I843], t. I, p.4I4-445). Elle consiste en: I) une inductiongénéralisante afin d'établir une hypothèse, 2) un raisonnement, 3) une vérification (ibid., p. 5 10). La première étape pose le problème de savoir si les régularités observées correspondent à des lois identiques à celles de la physique ou s'il s'agit de lois tendancielles (Zouboulakis, 1993) qui impliqueraient que les résultats obtenus ne sont pas toujours vrais. En économie pure, Pareto évite ce biais en retenant l'hypothèse que les agents économiques sont parfaitement rationnels. Dès lors, le cadre de l'économie pure est assimilable à celui que Popper retient pour cette science. Il s'agit de «construire un modèle à partir du postulat d'une rationalité totale de la part de tous les individus en jeu, et à estimer la déviation du comportement effectif des gens par rapport au modèle de comportement, en utilisant ce dernier comme une sorte de coordonnée zéro » (Popper, 1988 [1944-1945], p. I77-178). L'hypothèse d'actions logiques constitue ainsi, la majeure d'un explicans à laquelle sont adjointes des mineures qui spécifient une ou plusieurs situations particulières. Pareto en déduit un théorème précisant l'optimum atteint pour chaque situation à expliquer (explicandum). Ce résultat est testable indépendamment des hypothèses sur lesquelles il repose. Confirmant cette posture, il traite tour à tour, dans Manuel (Pareto, I966 [1906], p. I65-168 et p. 343-379) : des marchés de concurrence pure, des monopoles et oligopoles puis des situations où l'État est chargé d'optimiser la production. 
Pour autant, Pareto abandonne cette méthode lorsqu'il étudie les phénomènes sociologiques à partir des interactions qu'il décrit entre les catégories de résidus, de dérivations, d'intérêts et d'hétérogénéité sociale. L'identification de ces interactions, observables historiquement, lui permet de mettre en évidence l'existence de lois tendancielles où la rupture de divers moments machiavéliens explique le passage d'un équilibre social à un autre. Les lois de la sociologie parétienne sont le résultat d’inductions généralisantes qui font qu'elles ne peuvent pas être assimilées à des lois universelles et considérées comme vraies au même titre que celles de la physique. Dès lors, deux options s'offrent à nous pour circonscrire la méthodologie de Pareto en sociologie.

La première consiste à retenir qu'il se réfère au modèle déductif concret de Mill en raison de la manière foncièrement inductive dont il établit les prémisses de ses raisonnements. La compilation d'exemples empruntés à l'actualité, aux historiens grecs ou romains et à Machiavel témoigne de cette tendance. Ces lois sont obtenues par induction pour définir un explicans et dépendent de conditions particulières qui leur confèrent un caractère tendanciel. Pareto rejoint Mill pour qui, en sociologie, il faut rechercher «les lois qui déterminent les circonstances générales elles-mêmes. Il s’agit de découvrir, non pas quel sera l'effet d'une cause donnée dans certains états de la société, mais quels sont les causes qui les produisent et les phénomènes qui caractérisent les états de la société en général (Mill, 1988 [1843], t. 2, p. 509). Or, pour Mill il est difficile d’appliquer la méthode déductive concrète à la sociologie (ibid., p. 489-49I) parce que de nombreuses causes sont à l'origine d'un phénomène. Mill invite alors au raisonnement abductif en indiquant que la méthode déductive concrète peut être sauvée en sociologie si les résultats obtenus sont vérifiés.

Ce remède consiste dans le procédé que, sous le nom de vérification, nous avons signalé comme la troisième partie constituante et essentielle de la méthode déductive, et qui n'est autre que la comparaison des conclusions du raisonnement, soit avec les phénomènes concrets mêmes, soit avec leurs lois empiriques quand on peut en obtenir. Le fondement de notre confiance, dans la science déductive concrète, n'est pas le raisonnement a priori même, mais l'accord de ses résultats avec ceux de l'observation a posteriori (ibid., p. 490). 
La seconde appréciation de la méthodologie de Pareto en sociologie consiste à admettre qu'il recourt à la «méthode déductive inverse ou historique » que Mill préconise pour cette discipline (ibid., p. 49I). Pareto propose en effet souvent de remonter d'une proposition particulière à une proposition générale dont la première peut être déduite. Il propose aussi de remonter d'une proposition particulière, ou d'un fait historique, à un autre fait qui en exprime la cause. Dans le premier cas, il recourt à un raisonnement régressif, dans le second, à un raisonnement abductif (Bouvier, 2009), c'est-à-dire à la méthode déductive inverse. Celle-ci pose problème dans la mesure où il s'agit de renforcer une loi par des vérifications portant sur les résultats qui en sont déduits, de sorte que Pareto développerait un raisonnement qui repose sur la forme abductive du modus ponens.

Pareto opte certainement pour une position intermédiaire entre modèle « déductif concret» et «déductif inverse » pour trois raisons principales.

Premièrement, parce qu'il s'emploie à dégager des lois tendancielles en sociologie à partir d'inductions comme le veut la méthode déductive concrète. Il s'engage aussi sur le terrain de la méthode déductive inverse lorsqu'il indique établir les lois de la dynamique sociale comme causes des états particuliers des équilibres sociaux qui sont constatés. Les uniformités sont ici révélées par l'ensemble des faits historiques de sorte que la méthode déductive inverse, éminemment inductive, implique de ramener les lois de la sociologie à des lois tendancielles. À cet égard, Mill, comme le souligne Popper, considère que la forme tendancielle des lois de la sociologie n'est pas critiquable en soi si ces lois peuvent être garanties par celles d'une autre science. Ce principe est repris en effet par Popper pour qui il ne faut « jamais introduire de généralisations dans les sciences sociales à partir de l'histoire, à moins d'indiquer des raisons suffisantes pour le faire, c'est-à-dire en les déduisant de certaines lois naturelles vérifiables qui peuvent être établies de façon indépendante» (Popper, 1988 [1944-1945], p. 152). Or, c'est précisément sur ce terrain que Pareto tente de s'engager. 
Deuxièmement, parce que Pareto indique, précisément en ce qui concerne le principe précédent, que les lois de la psychologie permettraient d'expliquer les comportements qui résultent des résidus, dérivations, intérêts et hétérogénéités ou circulation sociale même s'il précise vouloir seulement considérer leurs effets apparents (Bouvier, I999). Pareto nous prouve ainsi qu'il a perçu la difficulté mais ne l’a pas résolue.

Les actions logiques sont, au moins dans leur partie principale, le résultat d'un raisonnement; les actions non-logiques proviennent principalement d'un certain état psychique : sentiments, subconscience, etc. C'est à la psychologie à s'occuper de cet état psychique. Dans notre étude, nous partons de cet état de fait, sans vouloir remonter plus haut (Pareto, 1968 [1916], p. 74).

Troisièmement, parce que notre auteur use largement du principe de l'affirmation du conséquent (soit de la version abductive du modus ponens) pour formuler ses explications, ce qui pose problème quant à leur validité du point de vue strictement logique.

Le Traité revêt donc un caractère profondément inductif, que Pareto reconnaît même s'il tente de convaincre ses lecteurs que sa démarche serait, en sociologie, largement déductive'. Le problème est délicat parce qu’il remet en cause le souhait formulé par Pareto de ne pas séparer l'économie de la sociologie de manière à en réaliser la synthèse. Or, les figures de logique proposées pour traiter de la sociologie ne correspondent pas forcément à celles retenues pour l'économie pure de sorte que la synthèse des résultats de ces deux disciplines peut être mise en doute 2 . Le Pareto du Cours (1964 [1896-1897]) ou du Manuel (1966 [1906]) laisse en effet une place importante à l'inductif pour fonder les hypothèses sur lesquelles un raisonnement est construit. Il offre une place plus importante encore à la déduction ou au raisonnement nomologique pour établir les théorèmes de l'économie pure à partir de l'hypothèse de rationalité

I Ce thème est source de débats (Bouvier, 2009). Pour un économiste comme Bruni (2000), la méthode de Pareto est largement expérimentale ou inductive. Pour Cressanti (1985), elle est largement déductive.

2 Nombre de sociologues tendent à contourner cette difficulté en insistant sur la séparation entre actions logiques et non-logiques comme critère de démarcation entre l'économie et la sociologie (Valade, 1990; Boudon, 2000, 2013). 
à laquelle correspondent les actions logiques et qui peut s'interpréter comme un a priori pertinent. Pareto cherche ensuite confirmation des théorèmes obtenus en les confrontant à ce qu'il constate en économie appliquée.

Un bref exemple du passage de l'économie pure à l'économie appliquée peut illustrer cette remarque. Il a trait à la démonstration du théorème des productivités marginales à laquelle Pareto prend part à côté de Walras et Barone. Selon ce théorème, en libre concurrence, chaque facteur est rémunéré, à l'équilibre, au prorata de sa productivité marginale. Or, ce résultat, que Pareto critique mais dont il reprend la démonstration (Ragni, 2000), entre en contradiction avec la loi éponyme qu'il propose selon laquelle $80 \%$ des richesses ou de la production sont détenues par $20 \%$ d'une population. Pareto cherchera, par complexification croissante, à rendre compte de ces observations à partir des catégories de sa sociologie et des jeux de pouvoir entre les élites et la masse des individus de manière à expliquer une telle répartition des richesses. Cet exemple met en évidence une rupture de méthode dès lors qu'il s'agit à la fois d'infirmer (ou de vérifier) un théorème d'économie pure et d'établir une loi (celle des « 80/20») par induction ou d'en proposer une explication à partir de catégories supportant elles-mêmes des lois tendancielles propres à la sociologie. En d'autres termes, le problème est ici de conserver un théorème d'économie pure tout en constatant qu'il n'est pas forcément vérifié, puis d'expliquer ce qui est constaté à partir de lois plus larges, propres à la sociologie, qui elles-mêmes ne participent en rien aux fondements du théorème.

Ainsi, même si Pareto indique appliquer la méthode logico-expérimentale à l'économie et à la sociologie, il convient encore d'apprécier dans quelle mesure il y parvient et d'observer comment les raisonnements de sa sociologie sont fondés. Les principes qui prévalent à la rupture du moment machiavélien permettent justement d'éclairer cette difficulté. 


\subsection{RUPTURE DU MOMENT MACHIAVÉLIEN ET LIMITE DE LA MÉTHODE PARÉTIENNE}

Deux exemples peuvent illustrer le rôle de la rupture du moment machiavélien dans la dynamique de l'équilibre social. Ils témoignent des limites de la méthodologie parétienne.

Le premier a trait à l'alternative libre-échange/protectionnisme. Pareto démontre en économie pure la supériorité du libre-échange sur le protectionnisme et cherche ensuite à confirmer ce résultat en mettant statistiquement en évidence les conséquences néfastes de l'augmentation des tarifs douaniers italiens imposés par les lois de I887 (Pareto, I992 [I89I], I892, I893). Constatant l'insuffisance d'une démonstration mathématique pour que le libre-échange s'impose, il propose d'expliquer la succession des régimes libre-échangiste et protectionniste à partir des luttes que se livrent certaines élites. Cette analyse, récurrente dans le Traité (Aron, 1967; Steiner, 1995; Ragni, 2012), est illustrée par un schéma où les interactions entre résidus, dérivations, intérêts et hétérogénéité sociale sont examinées selon trois relations principales et trois relations secondaires explicitant la lutte entre élites favorables au protectionnisme ou au libreéchange (Pareto, 1968 [1916], p. 77-80).

Pareto nous explique que les intérêts des acteurs sociaux sont le résultat des interactions entre résidus de persistance des agrégats et d'instinct de combinaisons qui génèrent des actions non-logiques opposées selon que ces acteurs appartiennent à une élite qui aura intérêt à défendre le libre-échange ou le protectionnisme. Ces actions, et les résidus qui en sont à l'origine, génèrent deux relations principales qui émanent de l' «état psychologique des agents » et que Pareto qualifie d'Élément $A$.

La première relation concerne l'exercice pratique du libre-échange ou du protectionnisme qui est qualifiée d’Élément B. Il peut aussi s'agir d’un argumentaire favorisant la promotion de règles de droit expliquant pourquoi ces formes de commerce se sont succédé selon les époques. Les résidus de persistance des agrégats et d'instincts de combinaison ont pour rôles de favoriser l'organisation de ces institutions dont la fonction est de garantir les intérêts des élites qui en sont porteurs; l'élite munie des résidus les mieux adaptés finissant par imposer ses objectifs. On retrouve ici les circonstances qui prévalent aux oppositions entre les renards et 
les loups décrites par Machiavel. Les premiers recourent à la ruse et les seconds à la force selon qu'ils seront porteurs de résidus d'instinct de combinaisons ou de persistance des agrégats. L'important pour Pareto est de montrer que la démonstration d'un théorème affirmant la supériorité du libre-échange n’est en rien suffisante pour expliquer les raisons pour lesquelles le protectionnisme perdure ou au contraire cède la place au libre-échange. Le lien entre théorème démontré et intérêt est donc secondaire pour Pareto. C'est davantage, selon lui, la confrontation entre les actions non-logiques générées par des résidus opposés et supportés par des dérivations pertinentes qui permet à certaines élites de convaincre le plus grand nombre d’adopter le protectionnisme ou le libre-échange selon les circonstances.

La seconde relation exprime l'opposition des élites porteuses des résidus précédents afin de promouvoir la théorie pure du libre-échange ou celle du protectionnisme. L'une ou l'autre de ces théories constitue l'Élément $C$ de l'explication. Pour Pareto, qu'une théorie soit établie mathématiquement et réponde aux principes qui prévalent aux actions logiques ne garantit nullement qu'elle advienne dans les faits. C'est donc les rapports de forces entre élites, relayés par une argumentation efficace, qui expliquent que le protectionnisme ou le libre-échange adviennent comme résultat d’une série de ruptures du moment machiavélien.

Enfin, Pareto met en évidence comment les actions concrètes d'échanges commerciaux, adossées au libre-échange ou au protectionnisme - Élément D - résultent des états psychiques des agents ou de leurs comportements de groupe supportés par les résidus précédents et qui émanent de l'Élément $A$. Dès lors, l’adoption des institutions du libre-échange ou du protectionnisme - Élément B - résultent dans une moindre mesure des théories pures qui pourraient les justifier - Élément $C$ - ou des pratiques effectives de commerce - Élément D.

Les états de l'histoire où les doctrines du libre-échange et du protectionnisme se succèdent constituent autant d'instants où l'équilibre entre virtù et fortuna est rompu du fait d'institutions qui se révèlent insuffisamment solides (ordine). L'ordre établi en matière de commerce international ne peut, en effet, qu'être instable dans la mesure où il dépend de résidus incapables de s'imposer à long terme. Cette dialectique peut se traduire, dans la terminologie de Machiavel, comme une impossibilité pour la virtù à toujours résister aux injonctions de la 
fortuna. En effet, le plus souvent les élites qui se seront imposées en recourant aux résidus de persistance des agrégats seront, dans un deuxième temps, davantage enclins à recourir aux résidus d'instinct de combinaisons. Ils préféreront alors user de la ruse plutôt que de la force pour faire en sorte que le protectionnisme ou le libreéchange perdure. Aussi, une élite capable de percevoir le moment de saisir la fortuna, grâce à la virtù qui la caractérise, sera à même d'induire un renversement entre libre-échange et protectionnisme. En termes parétiens, les élites favorables au libre-échange, à un instant de l'histoire, seront de plus en plus marquées par des résidus d'instinct de combinaisons exprimant des comportements de ruse tels que Machiavel les prête aux renards. Ces mêmes élites se révéleront incapables de résister, dans certaines circonstances, aux résidus de persistance des agrégats qui engendrent souvent les comportements de force que Machiavel prête aux loups. Dans cette éventualité, l'élite favorable au protectionnisme se montrera capable d’activer des arguments pseudo-logiques suffisants afin d'imposer ses intérêts.

Dans le cas le plus probable où les actions non-logiques guident les acteurs sociaux, se sont donc bien les résidus et les dérivations qui jouent le rôle principal puisqu'elles renvoient aux ruses auxquelles un groupe d'agents recourt pour convaincre d'adopter la théorie du commerce qui lui convient. Il s'agit d'un mécanisme de lutte des forts contre les faibles qui place au second plan les démonstrations d'économie pure.

Un second exemple de l'importance du moment machiavélien dans la sociologie de Pareto apparait au chapitre du Traité consacré à la forme générale de la société à propos de la différence entre «maximum d'ophélimité pour une collectivité en économie ( Pareto, 1968 [1916], p. I338-134I) et «maximum d'utilité pour une collectivité en sociologie» (ibid., p. I34I-I345). Là aussi, c'est davantage les luttes entre élites qui expliquent pourquoi l'optimum d'une collectivité en sociologie, favorable à certaines d'entre elles, advient et pourquoi il ne peut pas être défini comme un optimum pour une collectivité en économie (Busino, 1968; Passeron, 1995). C'est la portée de l’applicabilité du concept d’optimum économique parétien qui est limitée par l'auteur lui-même. En économie, il est impossible de comparer inter-individuellement les utilités alors qu'une autorité publique peut comparer les utilités d'une collectivité. En sociologie, Pareto précise en effet que cela se fait 
"plus ou moins bien, souvent mal, très mal, et l'on dit que l'autorité publique doit s'arrêter au point à partir duquel, en continuant, elle ne procure aucun "avantage" à toute la collectivité; qu'elle ne doit pas infliger de souffrances "inutiles" à la collectivité entière ni à une partie de la collectivité» (Pareto, I968 [1916], p. I343).

En revanche l'ophélimité d'une collectivité n'existe pas en économie. En d'autres termes, une autorité publique (élite) est capable de proposer (d'imposer) certains choix à un collectif à l'avantage de certains groupes (et au détriment d'autres) alors que cela est impossible en économie parce qu’on ne peut pas considérer une collectivité comme une personne si l'on veut respecter le principe de l'optimalité parétienne: «En économie pure on ne peut pas considérer une collectivité comme une personne; en sociologie, on peut considérer une collectivité, sinon comme une personne, au moins comme une unité » (ibid.). C'est parce qu'il se heurte à l'impossibilité d'expliquer, par un modèle où la rationalité des actions logiques prévaut, comment certains optima collectifs sont atteints que Pareto en traite à partir des catégories de sa sociologie. En se référant à Machiavel, Pareto (ibid., p. I254-I255) explicite les raisons qui sont à l'origine d'actions non-logiques dont le but est de permettre à une élite de défendre une forme institutionnelle qui lui assure de se maintenir au pouvoir et, en même temps, d’atteindre un optimum social qui lui soit avantageux.

Il met en évidence comment les élites caractérisées par certains résidus d'instinct de combinaisons ou de persistance des agrégats accèdent au pouvoir ou s'y maintiennent selon que la masse des dominés est elle-même caractérisée ou non par l'un ou l'autre de ces résidus. Il précise que s'ils sont disposés à recourir à la force quand

des chefs sont capables de les y conduire les gouvernés accèderont au pouvoir si les gouvernants sont mus par trop de sentiments humanitaires (résidus de rapport à la sociabilité) et pas suffisamment de résidus d'instinct de combinaison de sorte qu'une aristocratie humanitaire gouvernante est certainement la forme institutionnelle la plus instable du pouvoir (ibid., p. I387).

Une telle situation est pour Machiavel propice aux affres de la fortuna et à la violence des loups si ceux qui sont au pouvoir ne savent pas y répondre. En revanche, Pareto précise qu’il est difficile de déposséder une élite du pouvoir lorsqu’elle possède majoritairement des résidus d'instinct de combinaison qui la rendent adroite à 
faire usage de la ruse ou de la corruption. On retrouve ici, une fois encore, les comportements que Machiavel prête aux renards (ibid., p. IoI9). Il faut néanmoins que cette élite soit capable de sássurer les services de ceux qui possèdent les mêmes qualités afin qu'ils ne cherchent pas à s'emparer eux-mêmes du pouvoir en entrâ̂nant ceux capables de recourir à la violence (ibid., p. 1387). Les renards de Machiavel doivent ici sarranger de la force des lions pour répondre à celle des loups.

Pareto montre ainsi que si les résidus d'instinct de combinaisons caractérisent un nombre suffisant de membres de l'élite au pouvoir et que suffisamment de résidus de persistance des agrégats caractérisent ceux de la classe gouvernée, le régime politique en vigueur présentera une forte stabilité (ibid., p. I388). A contrario, en référence à la ruse des renards contre la violence des loups, une situation inverse sera propice à la rupture de l'équilibre social (entre virtù et fortuna) et à un renversement institutionnel au détriment de l'élite au pouvoir.

L’accumulation d'éléments supérieurs dans les classes inférieures et, vice versa, d'éléments inférieurs dans les classes supérieures, est une cause puissante de perturbations de l'équilibre [...] Les révolutions se produisent parce que - soit à cause du ralentissement de la circulation de l'élite, soit pour une autre cause - des éléments de qualité inférieure sáccumulent dans les couches supérieures. Ces éléments ne possèdent plus les résidus capables de les maintenir au pouvoir, et ils évitent de faire usage de la force; tandis que, dans les couches inférieures, se développent des éléments de qualité supérieure, qui possèdent les résidus nécessaires pour gouverner, et qui sont disposés à faire usage de la force (ibid., I304-I305).

Parmi les effets que provoque le changement de proportion de résidus, de l'instinct des combinaisons et de la persistance des agrégats dans la classe gouvernante (§ 222I) il faut prendre garde à ceux qui peuvent affaiblir la résistance de cette classe, en lutte avec la classe gouvernée. [...] Mais pour gouverner, il faut la force; et à mesure que les résidus de l'instinct des combinaisons se développent et que ceux de la persistance des agrégats s'atrophient chez les gouvernants, ceux-ci deviennent toujours moins capables d'user de la force. Nous avons donc un équilibre instable, et des révolutions se produisent (ibid., p.I424-I425).

Ou encore en ce qui concerne les luttes entre partis politiques : 
Si les concessions faites aux partis extrêmes n’ont guère été efficaces par le passé, elles le deviendront de moins en moins à l'avenir, car, ainsi que l'avait déjà observé Machiavel, avec une profonde connaissance de la nature humaine, les concessions dont on peut croire que la cause est la crainte ou la faiblesse se retournent toujours contre ceux qui les font (Pareto, 1904, p. 244).

Si Pareto développe maints exemples de rupture de l'équilibre social, la méthodologie à laquelle il recourt pour en déduire une explication générale présente trois inconvénients. Premièrement, elle repose sur un raisonnement inductif critiquable si l'objectif est de mettre à jour des lois universelles de la dynamique de l'équilibre social comme il le prétend. Deuxièmement, il recourt souvent à des raisonnements abductifs ou régressifs qui ne permettent pas de valider les démonstrations qu'il avance dès lors que de tels raisonnements constituent des figures de logique non valides. Troisièmement, à aucun moment, il n'indique pourquoi certains résultats d'économie pure doivent être conservés dès lors que, selon les circonstances, ils se révéleront incompatibles avec son explication de la dynamique sociale. Cette troisième difficulté est quasiment impossible à résoudre sauf à envisager deux éventualités comme Pareto le propose : soit les démonstrations d'économie pure, dès lors qu'elles reposent sur l'hypothèse d’actions logiques, constituent des approximations premières dont les explications en sociologie peuvent ensuite se passer; soit, et cela conforte la remarque précédente, ces démonstrations n'influencent quasiment pas les modalités que Pareto retient pour expliquer la dynamique sociale ou l'émergence d'institutions parce que ces explications dépendent essentiellement des résidus, des dérivations et donc des actions non-logiques. Les deux premiers inconvénients peuvent être illustrés en reformulant la manière dont Pareto traite du maintien au pouvoir d'une élite en comparant raisonnement déductif et raisonnement abductif. Un raisonnement déductif devrait vérifier la figure du modus ponens suivante :

- p : «une élite A, pour gouverner une institution républicaine, doit posséder suffisamment de résidus de persistance des agrégats par rapport à ceux d'instinct de combinaisons »;

- p': «la masse des gouvernés possède suffisamment de résidus de persistance des agrégats »; 
- p": «une élite pour se maintenir au pouvoir doit être capable de se garantir les services de leaders appartenant à la masse des gouvernés et qui possèdent des résidus de persistance des agrégats »;

- si $p^{\wedge} p^{\prime \wedge} p ” \rightarrow q:$ «une institution républicaine favorable à l'élite $A$ au pouvoir perdure».

On déduit l'explanandum : q vrai.

Ce raisonnement est valide mais présente l'inconvénient de s'appliquer à toute forme de régime politique. En d'autres termes, il convient certainement de poser une série de clauses ad hoc pour circonscrire les situations particulières où une institution républicaine perdure de sorte que la loi proposée est une loi «non stricte». Par ailleurs l'explanandum q peut être infirmé comme le veut le modus tollens de sorte que l'explication devrait être rejetée ou considérée comme admissible sous la contrainte de spécifier certaines hypothèses ad hoc. Enfin, si l'on cherche à vérifier les prémisses par l'expérience il est difficile d'admettre que l'on puisse évaluer $p$, p' ou p".

Pareto recourt certainement davantage au raisonnement abductif qui peut être illustré de la manière suivante :

- $p$ : «une élite A pour gouverner une institution républicaine doit posséder suffisamment de résidus de persistance des agrégats par rapport à ceux d'instinct de combinaisons » ;

- p': «la masse des gouvernés possède suffisamment de résidus de persistance des agrégats »;

- p": «une élite pour se maintenir au pouvoir doit être capable de se garantir les services de leaders appartenant à la masse des gouvernés et qui possèdent des résidus de persistance des agrégats »;

- si $p^{\wedge} p^{\wedge} p^{\prime \prime} \rightarrow q:$ «une institution républicaine favorable à l'élite $A$ au pouvoir perdure »;

- q: un exemple historique montre qu'«une institution républicaine favorable à l'élite $\mathrm{A}$ au pouvoir perdure».

On déduit l'explanandum : q vrai. 
Ce raisonnement correspond assez bien aux propos de Pareto qui trouve toujours dans l'histoire un exemple confirmant ce qu'il cherche à montrer. Enfin, les observations qui portent sur le fait qu'une élite (ou une masse) détiendrait plus ou moins de certains résidus par rapport à tel autre permet à Pareto d'envisager de manière ad hoc toutes les situations qui pourraient advenir dans l'histoire politique ou sociale de sorte que son raisonnement fonctionne, en quelque sorte, «à chaque fois ». En outre, ce raisonnement présente l'inconvénient de ne pas être logiquement valide.

\section{CONCLUSION}

Les constats précédents résultent des intentions de Pareto comme il le confirme dans le discours de son jubilé :

arrivé à un certain point de mes recherches d'économie politique, je me trouvais dans une impasse. Je voyais la réalité expérimentale et ne pouvais l'atteindre. Plusieurs obstacles m'arrêtaient: entre autres la mutuelle dépendance des phénomènes sociaux; laquelle ne permet pas d'isoler entièrement l'étude des différents genres de ces phénomènes, et qui s'oppose à ce que l'une d'elle puisse progresser indéfiniment si elle demeure privée de l'aide des autres (Pareto, 1917, p. 67-68).

À la suite de ces propos, Pareto envisage trois moyens de résoudre cette difficulté : I) rejeter entièrement la science économique; 2) ne pas recourir à la méthode expérimentale et décrire ce qui devrait être et non ce qui est ; 3) considérer que le manque de concordance entre les théories et les faits résulte de leur traitement séparé par plusieurs disciplines. Dans ce dernier cas il admet «que ce que l'on nomme lois, dans les sciences expérimentales, ne sont nullement des conséquences nécessaires: la science expérimentale ignore l’absolu; ce sont de simples notions d'uniformités, qui, constatées dans le passé, nous permettent de prévoir, avec la probabilité plus ou moins grande l’avenir» (ibid., p. 68).

Pareto ne rejette pas la science économique, il n'opte pas non plus pour la deuxième solution comme en témoigne les reproches qu'il adresse à Walras à propos de la recherche d'un idéal social (Pareto, 1975, p. 289-290). En revanche, les limites qu'il reconnait à sa méthode témoignent de son embarras. 
L'exemplification à laquelle il recourt le conduit à établir des lois tendancielles de sorte que ses raisonnements supposent que l'on puisse toujours constater pourquoi une situation institutionnelle spécifique advient ou non. Cette conclusion résulte de l'emploi de la version abductive du modus ponens. Si une telle démarche permet d'expliquer, «de manière non absolue» selon les termes de Pareto, l'évolution des équilibres sociaux, elle met également en évidence la difficulté de réaliser la synthèse entre des démonstrations déductives en économie pure et des explications qui reposent sur des lois tendancielles et sur le principe d’affirmation du conséquent en sociologie. La méthode des approximations et de synthèse présente alors la faiblesse de réduire à des cas hypothétiques les résultats d'économie pure sans qu'ils puissent être intégrés aux explications de l'évolution de l'équilibre social que Pareto propose en sociologie et pour lesquelles le principe de rupture du moment machiavélien tient une place essentielle.

Cette synthèse révèle une autre limite de la méthode des approximations successives, qui doit, d'une part, rendre compatibles des résultats qui ne le sont pas forcément, et d'autre part, conduire à considérer les résultats de l'économie comme des cas particuliers de la sociologie. On peut alors s'interroger sur la pertinence qu'il y a à conserver les résultats d'économie pure dans la mesure où soit ils ne constituent qu'une première approximation par rapport aux lois de la sociologie - et dans ce cas on ne voit pas comment leur synthèse peut être réalisée avec ceux de cette discipline -, soit ils ne jouent aucun rôle en sociologie - auquel cas il convient de les abandonner, ce que Pareto refuse.

\section{BIBLIOGRAPHIE}

ARON R., 1938-1940a, «Le machiavélisme de Machiavel », in ARON, 1995 (1993), p. 59-85.

-, 1938-1940b, «La comparaison de Machiavel et Pareto », in ARON, 1995 (1993), p. 86- 110.

-, 1938-1940c, «Pareto et le machiavélisme du Xxe siècle», in ARON, 1995 (1993), p. $110-122$.

-, 1938-1940d, « Machiavélisme et tyrannies », in ARON, 1995 (1993), p. 123-160. 
-, 1967, Les Étapes de la pensée sociologique, Paris, Gallimard.

-, 1995 (1993), Machiavel et les tyrannies modernes, Paris, Le Livre de Poche.

AUDIER S., 2005, Machiavel, conflit et liberté, Paris, Vrin-EHESS.

BERGÈS M., 2000, Machiavel, un penseur masqué?, Bruxelles, Éditions Complexes.

BOUDON R., 2000, «Vilfredo Pareto: Rationalité ou irrationalité des croyances», in Id. Études sur les sociologues classiques, Paris, PUF, t. II, p. 163-200.

-, 2013, «Les actions logiques et non-logiques selon Pareto », Revue européenne des sciences sociales, 51-2, p. 19-46.

BOUVIER A., 1999, «La théorie de l'équilibre social chez Pareto: une théorie paralléliste. Versant causal et versant intentionnel de l'équilibre social», Revue européenne des sciences sociales, 37-116, p. 245-258.

-, 2009, «Pareto: Une conception nominaliste et naturaliste atypique de la culture» [en ligne]: <http://germanistik.ch/publikationenliste.php>.

BRUNI L., 2002, Vilfredo Pareto and the Birth of Modern Microeconomics, Cheltenham, UK and Northampton (MA), E. Elgar.

BURNHAM J., 1949 (1943), Les Machiavéliens défenseurs de la liberté, Paris, Calmann-Lévy. BUSINO G., 1968, Introduction à une histoire de la sociologie de Pareto, Genève, Droz.

-, 1984, «Introduction», in PARETO, 1984, p. 7-62.

-, 1999, «L'actualité des travaux de Vilfredo Pareto», Revue européenne de sciences sociales, 37-116, p. 359-380.

-, 2008, «La science sociale de Vilfredo Pareto?», Revue européenne des sciences sociales, 46-140, p. 107-132.

COLLIN D., 2008, Comprendre Machiavel, Paris, Armand Colin.

COLONNA D'ISTRIA G., et FRAPET R., 1980, L'Art politique chez Machiavel: principes et méthode, Paris, Vrin.

CRESSATI C., 1985, «Vilfredo Pareto e John Stuart Mill», II Pensiero Politico, I8-I, p. 39-54.

GAILLE-NIKODIMOV M., 200I, «Machiavel au prisme du "moment machiavélien"», in SFEZ et SENELLART, 200I, p. 23I-239. 
HALBWACHS M., 1978 (19|8), «Le Traité de sociologie générale de M. Vilfredo Pareto», Revue européenne des sciences sociales, 16-43, p.91-103.

LANG A., 2003, «La dialectique de la fortune et de la virtù chez Machiavel», Archives de philosophie, 66-4, p. 649-662.

LEGRIS A., et RAGNI L., 1999, «Recouvrement du champ de l'économie dans l'œuvre de Pareto: une mise en perspective du désaccord avec Walras», Revue européenne des sciences sociales, 37-116, p. 325-346.

MACHIAVEL N., 1952, Guvres complètes, Paris, Gallimard, «Bibliothèque de la Pléiade».

MARCHIONATTI R., 1999, «The Methodological Foundation of Pure and Applied Economics in Pareto», Revue européenne des sciences sociales, 37-116, p. 277-294. - et GAMBINO E., 1997, «Pareto and Political Economy as a Science:

Methodological Revolution and Analytical Advances in Economic Theory in the 1890s», Journal of Political Economy, 105-6, p. 1322-1348.

MÉNISSIER T., 200I, «Qu'est-ce que la vertu républicaine? Quelques remarques sur l'interprétation de Machiavel dans Le Moment machiavélien», in SFEZ et SENELLART, 200I, p. 24I-246.

MILL J.-S., 1988 (1843), Système de logique déductive et inductive, Paris, Pierre Mardaga.

PARETO V., 1892, «Traités de commerce », in PARETO, 1984, p. 72-81.

-, 1893, «Les effets de la protection douanière en Italie», in PARETO, 1984, p. 82-88.

-, 1904, «Concession ou résistance», in PARETO, 1984, p. 242-245.

-, 1917 «Discours», in PARETO, 1975, p. 65-70.

-, 1964 (I896-1897), Cours d'économie politique, Fuvres complètes, t. I-II, Genève, Droz.

-, 1966 (1906), Manuel d'économie politique, Euvres complètes, t. VII, Genève, Droz.

-, 1966 (1898), Statistiques et économie mathématique, Fuvres complètes, t. VIII, Genève, Droz.

-, 1967 (1905-1918), Programme et sommaire du Cours de sociologie suivi de Mon Journal, Euvres complètes, t. XI, Genève, Droz.

-, 1968 (1916), Traité de sociologie générale, Euvres complètes, t. XII, Genève, Droz.

-, 1970 (1920), « Le cycle ploutocratique », in Id. La Transformation de la démocratie, Euvres complètes, t. XIII, Genève, Droz, p.49-58. 
-, 1975, Jubilé du Professeur V. Pareto, Fuvres complètes, t. XX, Genève, Droz.

-, 1976 (1918), «Économie expérimentale», in Id., Faits et Théories, Fuvres complètes, t. XXI, Genève, Droz, p. 626-643.

-, 1984, Mythes et Idéologies, Fuvres complètes, t. VI, Genève, Droz.

-, 1987 (I898), Comment se pose le problème de l'économie pure?, in Id., Marxisme et économie pure, Euvres complètes, t. IX, Genève, Droz, p. 102-109.

-, 1992 (|89|), Le Protectionnisme en Italie, ses résultats, in Id. Libre-échangisme, protectionnisme et socialisme, Euvres complètes, t. IV, Genève, Droz, p. 103-113.

PASSERON J-C., 1995, «Weber et Pareto : La rencontre de la rationalité dans l'analyses sociologique», in Le Modèle et l'Enquête. Les usages du principe de rationalité dans les sciences sociales, L.-A Gérard-Varet et J.-Cl. Passeron (dir.), Paris, EHESS, p. 38-I37.

PERRIN G., 1966, La Sociologie de Pareto, Paris, PUF.

POPPER K., 1988 (1944-1945), Misère de l'historicisme, Paris, Press Pocket.

POCOCK J.G.A. et BOROT L., 1975, The Machiavellian Moment, Florentine Political Thought and the Atlantic Republican Tradition, Princeton, Princeton University Press.

RAGNI L., 2000, «Enjeux de querelles et de justice sociale du théorème des productivités marginales», Revue d'économie politique, I10-1, p. 133-161.

-, 2012, «What Vilfredo Pareto Brought to the Knowledge Economy», in Hand Book of Economic Knowledge, N. Arena, A Festré et N. Lazaris (dir.), Cheltenham - Northampton (MA), E. Elgar, p. 23-48.

SFEZ G., 2003, Léo Strauss, lecteur de Machiavel. La modernité du mal, Paris, Ellipses. - et SENELLART M. (dir.), 200I, L'Enjeu Machiavel, Paris, PUF.

STEINER Ph., 1995, «Vilfredo Pareto et le protectionnisme: l'économie politique appliquée, la sociologie générale et quelques paradoxes», Revue économique, 46-5, p. $124 \mid-1262$.

STRAUSS L., 1982 (1958), Pensée sur Machiavel, Paris, Payot.

VALADE B., 2005, «Le thème élitaire dans l'œuvre de Vilfredo Pareto », Revue française d'histoire des idées politiques, 22-2, p. 5-15.

ZOUBOULAKIS M., 1993, La Science économique à la recherche de ses fondements, Paris, PUF. 


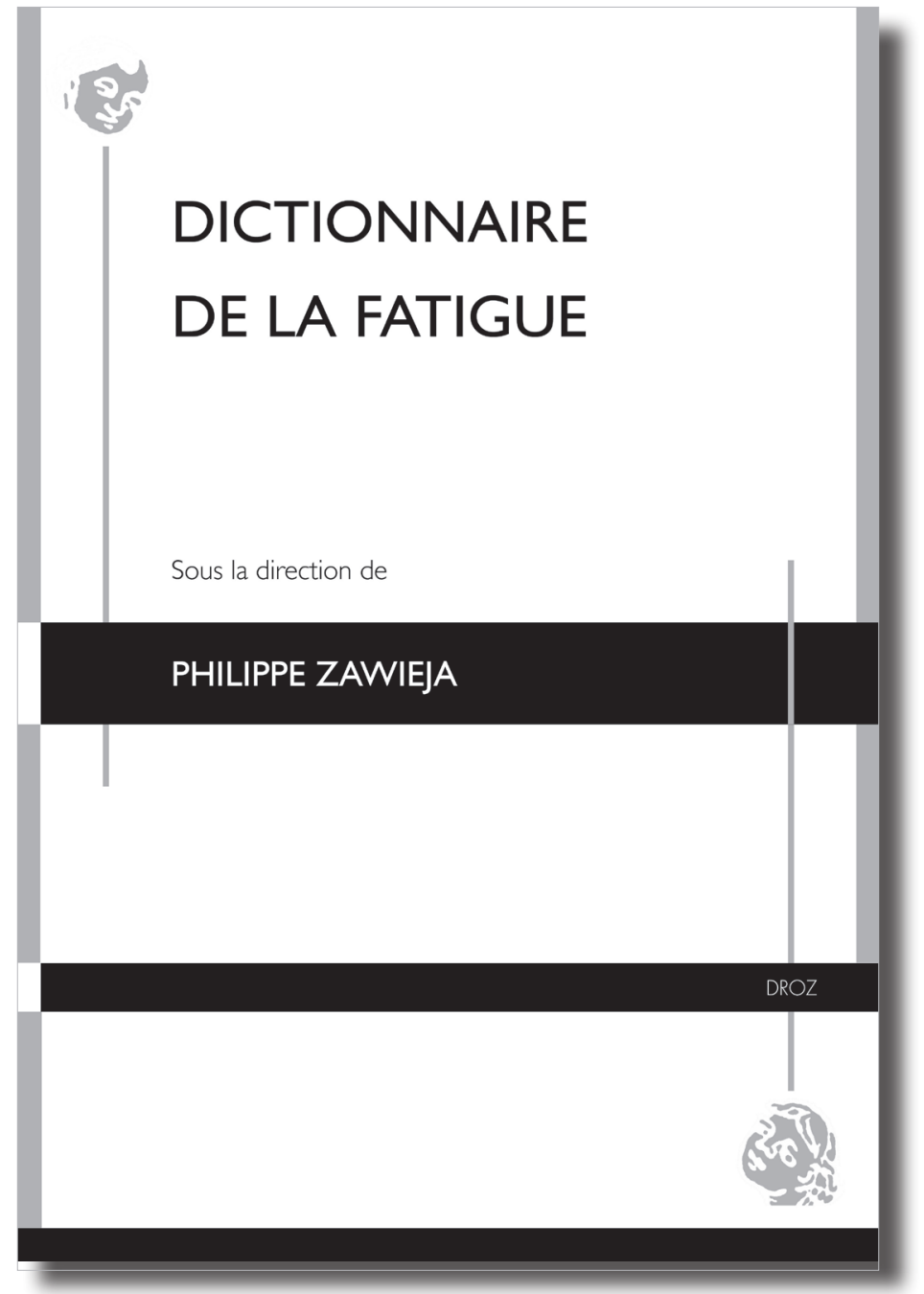

Librairie Droz, «Travaux des sciences sociales», n²19, 864 p. ISBN: 978-2-600-04713-5

Prix: $49,00 €$ TTC France 